\title{
LCR and AFD of the Products of Nakagami-m and Nakagami-m Squared Random Variables: Application to Wireless Communications Through Relays
}

Caslav Stefanovic ( $\nabla$ caslav.stefanovic@pr.ac.rs )

Faculty of Natural Sciences https://orcid.org/0000-0003-4385-3356

Ivan Milovanovic

Singidunum University

Mihajlo Stefanovic

University of Nis: Univerzitet u Nisu

\section{Research Article}

Keywords: AFD, AF relaying, FSO, LCR, second order statistical (SOS) performances, FSO

Posted Date: June 1st, 2021

DOl: https://doi.org/10.21203/rs.3.rs-380043/v1

License: (c) (1) This work is licensed under a Creative Commons Attribution 4.0 International License. Read Full License

Version of Record: A version of this preprint was published at Wireless Personal Communications on November 1st, 2021. See the published version at https://doi.org/10.1007/s11277-021-09258-6. 
Noname manuscript No.

(will be inserted by the editor)

\title{
LCR and AFD of the Products of Nakagami-m and Nakagami-m Squared Random Variables: Application to Wireless Communications Through Relays
}

\author{
Caslav Stefanovic • Ivan Milovanovic • \\ Mihajlo Stefanovic
}

Received: date / Accepted: date

\begin{abstract}
The paper considers level crossing rate (LCR) and average fade duration (AFD) of the product of independent and identically distributed (i.i.d) Nakagami-m (NM) and double NM squared (also known as gamma-gamma) random variables $(\mathrm{RVs})$. The derived statistical results are than related to the radio-frequency (RF) - free space optical (FSO), dual-hop (DH), amplifyand forward (AF) relaying system over non turbulent-induced-fading channels (nTIFCs) and turbulent-induced-fading channels (TIFCs). The obtained results of DH-AF, RF-FSO system over TIFCs and nTIFCs are numerically evaluated and graphically presented for all system model parameters.
\end{abstract}

Keywords AFD · AF relaying · FSO - LCR · second order statistical (SOS) performances $\cdot$ FSO

\section{Introduction}

Relaying wireless systems play an important role in wireless communications. The huge variety of technologies that includes relays are all radio-frequency (RF) relaying schemes, all free-space-optical (FSO) relaying schemes, mixed RF-FSO relaying schemes, millimeter-wave ( $\mathrm{mmW}$ )-FSO relaying schemes given in [1]-[4], respectively. In particular, amplify-and-forward (AF) relaying systems has been proposed for application in fronthaul/backhaul 5G systems

C. Stefanovic

Faculty of Natural Sciences, Universiy of Pristina, Serbia

E-mail: caslav.stefanovic@pr.ac.rs

I. Milovanovic

Singidunum University, Danijelova 32, 11000 Belgrade, Serbia E-mail: imilovanovic@singidunm.ac.rs

M. Stefanovic

Faculty of Electronic Engineering, University of Nis, Serbia E-mail: mihajlo.stefanovic@elfak.ni.ac.rs 
[5], vehicle-to-everything (V2X) communications [6]-[7], unmanned ariel vehicle (UAV) communications [8], device-to-device (D2D) communications [9], mobile-to-mobile (M2M) communications [10]-[13] and underwater opticalwireless (UOW) communications scenario [14]. FSO relaying enabled communications can ensure high capacity and wide bandwidth [15]. Moreover, FSO communications are 1.) cost effective, 2.) not sensitive to co-channel interference and 3.) spectrum license free that makes this type of technology a promising choice for future wireless systems. The main factor of FSO system performance degradation is caused by atmospheric turbulence (also known as scintillation). The weather conditions and pointing errors can result in an additional FSO system performance deterioration. The references [16]-[18] investigates RF-FSO relay systems while FSO-FSO relay systems are explored in [19]. The above-mentioned works consider the first-order statistics (FOS) of mixed RF-FSO relay systems. Besides the FOS, the second order statistics (SOS) such as average level crossing rate (LCR) and average fade duration (AFD) can more adequately characterize the time-variant fading channels. The LCR of gamma-gamma random variable (RV) is addressed in [20], while the LCR over Malaga TIFC is addressed in [21]. The LCR expression of the ratio of two gamma-gamma RVs is derived in [22]. Moreover, the SOS over log-normal TIFC of multi-hop FSO system including pointing errors are addressed in [23]. The SOS of V2V of mixed RF-FSO-RF over NM nTIFC and gamma-gamma TIFC are considered in [6]. The [24]-[25] gives some experimental results for SOS of FSO systems. The Nakagami-m (NM) RV can be used to model nTIFC in RF environments [26]-[28], while gamma-gamma (double squared NM) RV is the most often used to model the fading for FSO communications in moderate to strong TIFC environments [29]-[31]. This paper considers the SOS of the product of independent and identically distributed (i.i.d) NM RV and gamma-gamma RV (modeled as the product of two squared NM RVs). The derived statistical measures are directly applied to dual-hop (DH) AF relay RF-FSO communications over mixed nTIFC and TIFC. The derived integral expressions as well as closed form approximations for LCR and AFD are numerically evaluated and graphically presented in relation to DH AF RF-FSO system model set of parameters. To the best of author's knowledge there is no publications on the SOS of DH-AF mixed RF-FSO systems.

\section{System Model}

The cascaded, double and composite fading channels can be modeled as the product of RVs [9], [13], [23], [32]-[34]. On the other hand, interference limited environments can be modeled as the ratio of RVs [35]-[37]. Thus, the products and the ratios of RVs play an important role in the performance analysis of wireless communication systems. It has been shown by [38, Eq. 39] that the signal envelope at the output of AF relaying over nTIFC for fixed gain relays can be modeled as the product of N Rayleigh RVs. The FSO multi-hop AF 


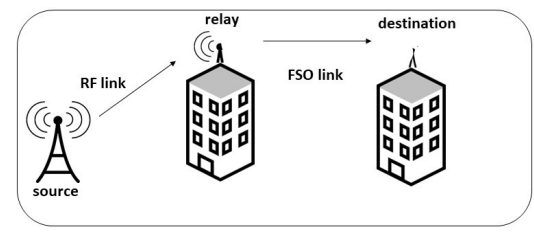

Fig. 1 Simplified scheme of RF-FSO dual-hop AF relaying

relaying over TIFC are considered as a product of log-normal RVs in [23, Eq. $13]$.

Thus, we model the fading signal envelope from source to destination, $z_{S D}$ of DH-AF relay (shown in Figure 1) as the product of the NM RV, $y_{N M 1}$ and gamma-gamma RV, $y_{G G}$ (modeled as the double NM squared i.i.d RVs) [26, Eq. (2.55)]:

$$
z_{S D}=\underbrace{y_{N M 1}}_{\text {Ihop }} \underbrace{y_{G G}}_{\text {I hop }}=\underbrace{y_{N M 1}}_{\text {Ihop }} \underbrace{y_{N M 2}^{2} y_{N M 3}^{2}}_{\text {IIhop }}
$$

where $y_{N M, i}$ are NM RVs, representing the signal envelopes over nTIFCs and TIFCs from source to destination, whose probability density functions (PDFs) in terms of fading severity parameters and average powers, denoted as $m_{N M, i}$ and $\omega_{N M, i}$ respectively, are:

$$
p_{Y_{N M}}\left(y_{N M}\right)=\frac{2\left(m_{N M, i} / \omega_{N M, i}\right)^{m_{N M, i}}}{\Gamma\left(m_{N M, i}\right)} y_{N M}^{2 m_{N M, i}-1} e^{-\frac{m_{N M, i}}{\omega_{N M, i}} y_{N M, i}^{2}}
$$

The PDFs parameters of the FSO NM squared RVs can be expressed as [29], [41]:

$$
\begin{gathered}
\omega_{N M 2}=\omega_{N M 3}=1 \\
\alpha_{G G}=m_{N M 2}=\left[\exp \left(\frac{0.49 \delta^{2}}{\left(1+0.18 d^{2}+0.56 \delta^{12 / 5}\right)^{7 / 6}}\right)-1\right]^{-1}, \\
\beta_{G G}=m_{N M 3}=\left[\exp \left(\frac{0.51 \delta^{2}\left(1+0.69 \delta^{12 / 5}\right)^{-5 / 6}}{\left(1+0.9 d^{2}+0.62 d^{2} \delta^{12 / 5}\right)^{5 / 6}}\right)-1\right]^{-1},
\end{gathered}
$$

where $\alpha_{G G}$ and $\beta_{G G}$ are FSO small-scale and large-scale atmospheric cell parameters, respectively, $\delta^{2}=0.5 C_{n}^{2} k_{F S O}^{7 / 6} L^{11 / 6}$ is the FSO Rytov variance 
and $d=\sqrt{k_{F S O} D_{F S O}^{2} / 4 L}$ is the FSO wave number. Moreover, $C_{n}^{2}$ is FSO refractive index, $k_{F S O}=2 \pi / \lambda$ is FSO wave-number ( $\lambda$-wavelength), $D_{F S O}$ is FSO receiver aperture diameter and $L$ is FSO distance between source and destination.

\section{Second Order Statistics (SOS)}

The level crossing rate (LCR) of the product of NM and double squared NM RV for a given threshold $z_{t h, S D}$ can be expressed through integration of the joint PDF of $z_{S D}$ and its first derivative $\dot{z}_{S D}[41]$,

$$
N_{z_{S D}}\left(z_{t h, S D}\right)=\int_{0}^{\infty} \dot{z}_{S D} p_{z_{S D} \dot{z}_{S D}}\left(z_{t h, S D} z_{S D}\right) d \dot{z}_{S D}
$$

which can be further expressed through some mathematical manipulations based on joint and conditional PDFs:

$$
\begin{aligned}
& p_{z_{S D} \dot{z}_{S D} Y_{N M 2} Y_{N M 3}}\left(z_{S D} \dot{z}_{S D} y_{N M 2} y_{N M 3}\right) \\
& =p_{\dot{z}_{S D} \mid z_{S D} Y_{N M 2} Y_{N M 3}}\left(\dot{z}_{S D} \mid z_{S D} y_{N M 2} y_{N M 3}\right) \\
& * p_{z_{S D} \mid Y_{N M 2} Y_{N M 3}}\left(z_{S D} y_{N M 2} y_{N M 3}\right) p_{Y_{N M 2}}\left(y_{N M 2}\right) p_{Y_{N M 3}}\left(y_{N M 3}\right)
\end{aligned}
$$

where, $p_{z_{S D} \mid Y_{N M 2} Y_{N M 3}}\left(z_{S D} y_{N M 2} y_{N M 3}\right)=\left|\frac{d y_{N M 1}}{d z_{S D}}\right| p_{Y_{N M 1}}\left(\frac{z_{S D}}{y_{N M 2}^{2} y_{N M 3}^{2}}\right)$

The variance of $\dot{z}_{S D}$, denoted as $\sigma_{\dot{z}_{S D}}^{2}$ is a zero mean Gaussian RP and after simple mathematical manipulation can be expressed through the variances of $\dot{Y}_{N M 1}, \dot{Y}_{N M 2}$ and $\dot{Y}_{N M 3}$, denoted as, respectively, $\sigma_{\dot{Y}_{N M 1}}^{2}, \sigma_{\dot{Y}_{N M 2}}^{2}$ and $\sigma_{\dot{Y}_{N M 3}}^{2}$ :

$$
\sigma_{\dot{z}_{S D}}^{2}=\frac{4 z_{S D}^{2}}{y_{N M 1}^{2}} \sigma_{\dot{Y}_{N M 1}}^{2}\left(1+\frac{4 z_{S D}^{2}}{y_{N M 2}^{4} y_{N M 3}^{6}} \frac{\sigma_{\dot{Y}_{N M 2}}^{2}}{\sigma_{\dot{Y}_{N M 1}}^{2}}+\frac{4 z_{S D}^{2}}{y_{N M 2}^{6} y_{N M 3}^{4}} \frac{\sigma_{\dot{Y}_{N M 3}}^{2}}{\sigma_{\dot{Y}_{N M 1}}^{2}}\right)
$$

After evaluating the integral,

$$
\int_{0}^{\infty} \dot{z}_{S D} p_{\dot{z}_{S D} \mid z_{S D} Y_{N M 2} Y_{N M 3}}\left(\dot{z}_{S D} \mid z_{S D} y_{N M 2} y_{N M 3}\right) d \dot{x}_{S D}=\frac{1}{\sqrt{2 \pi}} \sigma_{\dot{z}_{S D}}
$$

the $N_{z_{S D}}\left(z_{t h, S D}\right)$ can be expressed after some manipulations as:

$$
N_{Z_{S D}}\left(z_{t h, S D}\right)=\frac{8\left(m_{N M 1} / \omega_{N M 1}\right)^{m_{N M 1}} \alpha_{G G}^{\alpha_{G G}} \beta_{G G}^{\beta_{G G}} \sigma_{\dot{Y}_{N M 1}} z_{t h, S D}^{2 m_{N M 1}}}{\sqrt{2 \pi} \Gamma\left(m_{N M 1}\right) \Gamma\left(\alpha_{G G}\right) \Gamma\left(\beta_{G G}\right)} J_{1}
$$

where, 


$$
\begin{aligned}
& J_{1}=\int_{0}^{\infty} d y_{N N 2} \int_{0}^{\infty} \sqrt{\left(1+\frac{4 z_{S D}^{2}}{y_{N M 2}^{4} y_{N M 3}^{6}} \frac{\sigma_{\dot{Y}_{N M 2}}^{2}}{\sigma_{\dot{Y}_{N M 1}}^{2}}+\frac{4 z_{S D}^{2}}{y_{N M 2}^{6} y_{N M 3}^{4}} \frac{\sigma_{\dot{Y}_{N M 3}}^{2}}{\sigma_{\dot{Y}_{N M 1}}^{2}}\right)} \\
& * e^{-\frac{m_{N M 1}}{\omega_{N M 1}} \frac{z_{t h, S D}^{2}}{y_{N M 2}^{4} y_{N M 3}^{4}}-\alpha_{G G} y_{N M 1}^{2}-\beta_{G G} y_{N M 2}^{2}+\left(2 \alpha_{G G}-4 m_{N M 1}+1\right) \ln \left(y_{N M 2}\right)} \\
& * e^{\left(2 \beta_{G G}-4 m_{N M 1}+1\right) \ln \left(y_{N M 3}\right)} d y_{N M 3}
\end{aligned}
$$

The $J_{1}$, can be approximated by [38, Eq.I.3]:

$$
\begin{aligned}
& \int_{0}^{\infty} d y_{N M 2} \int_{0}^{\infty} g\left(y_{N M 2}, y_{N M 3}\right) e^{-\gamma f\left(y_{N M 1}, y_{N M 2}\right)} d y_{N M 3} \\
& \approx \frac{2 \pi}{\gamma} \frac{g\left(y_{N M 2}(0), y_{N M 3}(0)\right)}{\sqrt{\operatorname{det} B}} e^{-\gamma f\left(y_{N M 2}(0), y_{N M 3}(0)\right)}
\end{aligned}
$$

where, $y_{N M 2}(0)$ and $y_{N M 3}(0)$ are positive and real number values obtained from the defined set of equations,

$$
\frac{\partial f\left(y_{N M 2}(0), y_{N M 3}(0)\right)}{\partial y_{N M 2}(0)}=0, \frac{\partial f\left(y_{N M 2}(0), y_{N M 3}(0)\right)}{\partial y_{N M 3}(0)}=0
$$

and where $\mathrm{B}$ is,

$$
B=\left[\begin{array}{cc}
\frac{\partial^{2} f\left(y_{N M 2}(0), y_{N M 3}(0)\right)}{\partial y_{N M 2}(0)^{2}} & \frac{\partial^{2} f\left(y_{N M 2}(0), y_{N M 3}(0)\right)}{\partial y_{N M 2}(0) \partial y_{N 3}(0)} \\
\frac{\partial^{2} f\left(y_{N M 2}(0), y_{N M 3}(0)\right)}{\partial y_{N M 3}(0) \partial y_{N M 2}(0)} & \frac{\partial^{2} f\left(y_{N M 2}(0), y_{N M 3}(0)\right)}{\partial y_{N M 3}(0)^{2}}
\end{array}\right]
$$

The LCR can be approximated for the following choice of parameters and functions: $\gamma=1$,

$$
\begin{gathered}
g\left(y_{N M 2}, y_{N M 3}\right)=\sqrt{\left(1+\frac{4 z_{S D}^{2}}{y_{N M 2}^{4} y_{N M 3}^{6}} \frac{\sigma_{\dot{Y}_{N M 2}}^{2}}{\sigma_{\dot{Y}_{N M 1}}^{2}}+\frac{4 z_{S D}^{2}}{y_{N M 2}^{6} y_{N M 3}^{4}} \frac{\sigma_{\dot{Y}_{N M 3}}^{2}}{\sigma_{\dot{Y}_{N M 1}}^{2}}\right)} \\
f\left(y_{N M 1}, y_{N M 2}\right)=\frac{m_{N M 1}}{\omega_{N M 1}} \frac{z_{t h, S D}^{2}}{y_{N M 2}^{4} y_{N M 3}^{4}}+\alpha_{G G} y_{N M 1}^{2}+\beta_{G G} y_{N M 2}^{2} \\
-\left(2 \alpha_{G G}-4 m_{N M 1}+1\right) \ln \left(y_{N M 2}\right)-\left(2 \beta_{G G}-4 m_{N M 1}+1\right) \ln \left(y_{N M 3}\right)
\end{gathered}
$$

Finally, the closed form approximation of $N_{Z_{S D}}\left(z_{t h, S D}\right)$ becomes: 


$$
\begin{aligned}
& N_{Z_{S D}}\left(z_{t h, S D}\right) \approx \frac{16 \pi\left(m_{N M 1} / \omega_{N M 1}\right)^{m_{N M 1}} \alpha_{G G}^{\alpha_{G G}} \beta_{G G}^{\beta_{G G}} \sigma_{\dot{Y}_{N M 1}} z_{t h, S D}^{2 m_{N M 1}}}{\sqrt{2 \pi} \Gamma\left(m_{N M 1}\right) \Gamma\left(\alpha_{G G}\right) \Gamma\left(\beta_{G G}\right)} \\
& * \frac{\sqrt{\left(1+\frac{4 z_{S D}^{2}}{y_{N M 2}(0)^{4} y_{N M 3}(0)^{6}} \frac{\sigma_{\dot{Y}_{N M 2}}^{2}}{\sigma_{\dot{Y}_{N M 1}}^{2}}+\frac{4 z_{S D}^{2}}{y_{N M 2}(0)^{6} y_{N M 3}(0)^{4}} \frac{\sigma_{\dot{Y}_{N M 3}}^{2}}{\sigma_{\dot{Y}_{N M 1}}^{2}}\right.}}{\sqrt{\operatorname{det} B}} \\
& * e^{-\left(\frac{m_{N M 1}}{\omega_{N M 1}} \frac{z_{t h, S D}^{2}}{y_{N M 2}(0)^{4} y_{N M 3}^{4}(0)}+\alpha_{G G} y_{N M 1}^{2}(0)+\beta_{G G} y_{N M 2}^{2}(0)\right)} \\
& * e^{\left(2 \alpha_{G G}-4 m_{N M 1}+1\right) \ln \left(y_{N M 2}(0)\right)+\left(2 \beta_{G G}-4 m_{N M 1}+1\right) \ln \left(y_{N M 3}(0)\right)}
\end{aligned}
$$

The AFD can be then computed as [26], [39]:

$$
A F D\left(z_{t h, S D}\right)=\frac{F_{z_{S D}}\left(z_{t h, S D}\right)}{N_{Z_{S D}}\left(z_{t h, S D}\right)}
$$

The exact expression for cumulative distribution function (CDF) of the product two or more RVs can be derived from [26], [39]. The CDF of $z_{S D}$, denoted as $F_{z_{S D}}\left(z_{S D}\right)$ can be expressed as:

$$
F_{z_{S D}}\left(z_{S D}\right)=\int_{0}^{z_{S D}} p_{Z_{S D}}(s) d s
$$

where $p_{Z_{S D}}\left(z_{S D}\right)$ is the probability density function (PDF) at the output of DH-AF RF-FSO proposed model and can be further expressed as:

$$
\begin{aligned}
& p_{z_{S D}}\left(z_{S D}\right)=\int_{0}^{\infty} d y_{N M 2} \int_{0}^{\infty}\left|\frac{d y_{N M 1}}{d z_{S D}}\right| p_{Y_{N M 1}}\left(\frac{z_{S D}}{y_{N 2}^{2} y_{N 3}^{2}}\right) \\
& * p_{Y_{N M 2}}\left(y_{N M 2}\right) p_{Y_{N M 3}}\left(y_{N M 3}\right) d y_{N M 3}
\end{aligned}
$$

After substitution (20) in (19) and after using [40, Eq. (3.381.1)], [40, Eq. (8.352.1)] and [40, Eq. (3.471.9)], respectively for the case where $m_{N M 1}$ is integer number, $F_{z_{S D}}\left(z_{S D}\right)$ becomes:

$$
\begin{aligned}
& F_{z_{S D}}\left(z_{S D}\right)=\frac{4\left(\alpha_{G G}\right)^{\alpha_{G G}}\left(\beta_{G G}\right)^{\beta_{G G}}\left(\frac{m_{N M 1}}{\omega_{N M 1}}\right)^{m_{N M 1}}\left(m_{N M 1}-1\right) !}{\Gamma\left(m_{N M 1}\right) \Gamma\left(\alpha_{G G}\right) \Gamma\left(\beta_{G G}\right)} \\
& *\left(\frac{\Gamma\left(\alpha_{G G}\right) \Gamma\left(\beta_{G G}\right)}{4\left(\alpha_{G G}\right)^{\alpha_{G G}}\left(\beta_{G G}\right)^{\beta_{G G}}}\left(\frac{\omega_{N M 1}}{m_{N M 1}}\right)^{m_{N M 1}}-\sum_{k=0}^{m_{N M 1}-1} \frac{\left(\frac{m_{N M 1} z_{S D}^{2}}{\omega_{N M 1}}\right)^{k}}{k !} J_{2}\right)
\end{aligned}
$$

where $J_{2}$ in (21) is given:

$$
\begin{aligned}
& J_{2}=\int_{0}^{\infty} d y_{N M 2} \int_{0}^{\infty} y_{N M 2}^{2 m_{N M 2}-4 k-1} y_{N M 3}^{2 m_{N M 3}-4 k-1} \\
& * e^{-\left(\frac{m_{N M 1}}{\omega_{N M 1}} \frac{\left(z_{S D}\right)^{2}}{y_{N M 2}^{4} y_{N M 3}^{4}}+\alpha_{G G} y_{N M 2}^{2}+\beta_{G G} y_{N M 3}^{2}\right)} d y_{N M 3}
\end{aligned}
$$


The closed form approximation of $F_{z_{S D}}\left(z_{S D}\right)$ can be calculated with a help of (12)-(14) for the following choices of functions and parameters: $\gamma=1$, $g\left(y_{N M 2}, y_{N M 3}\right)=1$

$$
\begin{aligned}
& f\left(y_{N M 1}, y_{N M 2}\right)=\frac{m_{N M 1}}{\omega_{N M 1}} \frac{z_{t h, S D}^{2}}{y_{N M 2}^{4} y_{N M 3}^{4}}+\alpha_{G G} y_{N M 1}^{2}+\beta_{G G} y_{N M 2}^{2} \\
& -\left(2 \alpha_{G G}-4 k+1\right) \ln \left(y_{N M 2}\right)-\left(2 \beta_{G G}-4 k+1\right) \ln \left(y_{N M 3}\right)
\end{aligned}
$$

Finally, the closed form $F_{z_{S D}}\left(z_{S D}\right)$ is:

$$
\begin{aligned}
& F_{z_{S D}}\left(z_{S D}\right) \approx \frac{4\left(\alpha_{G G}\right)^{\alpha_{G G}}\left(\beta_{G G}\right)^{\beta_{G G}}\left(\frac{m_{N M 1}}{\omega_{N M 1}}\right)^{m_{N M 1}}\left(m_{N M 1}-1\right) !}{\Gamma\left(m_{N M 1}\right) \Gamma\left(\alpha_{G G}\right) \Gamma\left(\beta_{G G}\right)} \\
& *\left(\frac{\Gamma\left(\alpha_{G G}\right) \Gamma\left(\beta_{G G}\right)}{4\left(\alpha_{G G}\right)^{\alpha_{G G}}\left(\beta_{G G}\right)^{\beta_{G G}}}\left(\frac{\omega_{N M 1}}{m_{N M 1}}\right)^{m_{N M 1}}-\sum_{k=0}^{m_{N M 1}-1} \frac{\left(\frac{m_{N M 1} z_{S D}^{2}}{\omega_{N M 1}}\right)^{k}}{k !} J_{3}\right)
\end{aligned}
$$

where,

$$
\begin{aligned}
& J_{3} \approx \frac{2 \pi}{\operatorname{det}\left[\begin{array}{cc}
\frac{\partial^{2} f}{\partial y_{N M 2}(0)^{2}} & \frac{\partial^{2} f}{\partial y_{N M 2}(0) \partial y_{N 3}(0)} \\
\frac{\partial^{2} f}{\partial y_{N M 3}(0) \partial y_{N M 2}(0)} & \frac{\partial^{2} f}{\partial y_{N M 3}(0)^{2}}
\end{array}\right]} \\
& * e^{-\left(\frac{m_{N M 1}}{\left.\omega_{N M 1} \frac{z_{S D}^{2}}{y_{N M 2}(0)^{4} y_{N M 3}^{4}(0)}+\alpha_{G G} y_{N M 1}^{2}(0)+\beta_{G G} y_{N M 2}^{2}(0)\right)}\right.} \\
& * e^{\left(2 \alpha_{G G}-4 k+1\right) \ln \left(y_{N M 2}(0)\right)+\left(2 \beta_{G G}-4 k+1\right) \ln \left(y_{N M 3}(0)\right)}
\end{aligned}
$$

wheras, $f\left(y_{N M 1}, y_{N M 2}\right)$ for simpler notation is denoted as $f$.

\section{Numerical results}

The SOS are provided through LCR and AFD statistical measures. The variance of NM RV in (8) is expressed as $\sigma_{\dot{Y}_{N M 1}}^{2}=\pi^{2} f_{m N M 1}^{2} \frac{\omega_{N M 1}}{m_{N M 1}}$, where $f_{m}=$ $f_{m N M 1}$ is the maximum Doppler frequency. The variance of double NM squared $\mathrm{RV}, \sigma_{\dot{Y}_{G G}}^{2}$ in (8) is modeled as a zero mean Gaussian RV and expressed as, $\sigma_{\dot{Y}_{G G}}^{2}=f_{0}^{2} \pi^{2} \sigma_{G G}^{2}\left\langle Y_{G G}\right\rangle$, as has been introduced in [21, Eq. (13)]. Further, $\left\langle Y_{G G}\right\rangle=1$ for double NM squared RV [41] and $f_{0}=\frac{1}{\pi \tau_{0} \sqrt{2}}$ is the FSO quasi frequency, where $t_{0}=\frac{\sqrt{\lambda L}}{u t}$ is turbulence FSO correlation time, $\lambda$ is the FSO wavelength, $L$ is the FSO distance and $u t$ is the speed of wind directed towards the FSO part of the system [21].

Figure 2 shows that closed form $N_{Z_{S D}}\left(z_{t h, S D}\right)$ provided in (10) and presented for various RF-FSO sets of parameters $\left(f_{m}, \lambda\right)$, constant $u t=1 \mathrm{~m} / \mathrm{s}$ 


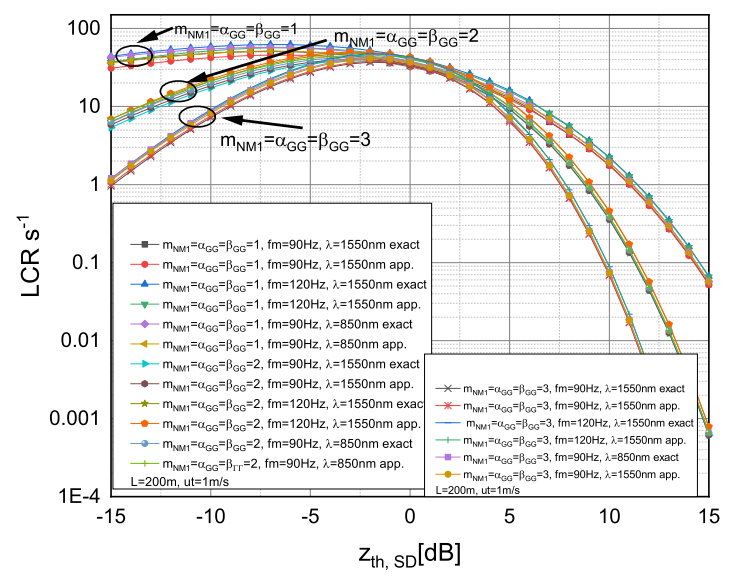

Fig. 2 Comparison of exact and approximated results for LCR for different system model parameters under different TIFC and nTIFC conditions

and $L=200 \mathrm{~m}$ and under different TIFC and nTIFC conditions, $\left(\alpha_{G G}=3\right.$, $\left.\beta_{G G}=3, m_{N M 1}=3\right),\left(\alpha_{G G}=2, \beta_{G G}=2, m_{N M 1}=2\right)$ and $\left(\alpha_{G G}=1\right.$, $\beta_{G G}=1, m_{N M 1}=1$ ) fits well with exact integral form solution provided in (17). Moreover, in the case when output signals experience TIFC and nTIFC conditions change that result in increase in nTIFC and TIFC severities, $N_{Z_{S D}}\left(z_{t h, S D}\right)$ decreases, as expected. The impact of the maximal Doppler frequency for $\left(f_{m}=90 \mathrm{~Hz}\right.$ and $\left.f_{m}=120 \mathrm{~Hz}\right)$ and FSO windows for $(\lambda=850 \mathrm{~nm}$, and $\lambda=1550 \mathrm{~nm})$ on $N_{Z_{S D}}\left(z_{t h, S D}\right)$ is then investigated. By increasing the FSO wavelength, the graphs slightly decrease. Contrary, by increasing observed $f_{m}$, the graphs slightly increase. Furthermore, it is obvious that nTIFC and TIFC parameters have stronger impact on $N_{Z_{S D}}\left(z_{t h, S D}\right)$ than $f_{m}$ and $\lambda$ which means that RF and FSO channel severity conditions have much stronger impact on $N_{Z_{S D}}\left(z_{t h, S D}\right)$.

The $\operatorname{AFD}\left(z_{t h, S D}\right)$ behavior is provided in Figure 3. It can be noticed that curves fitting between closed form approximate expression and exact integral analytical expression for observed system RF-FSO DH-AF parameters is achieved, especially for higher $z_{t h, S D} \mathrm{~dB}$ values. By increasing nTIFC and TIFC RF-FSO severities, $A F D\left(z_{t h, S D}\right)$ increases for lower dB output values while $A F D\left(z_{t h, S D}\right)$ decreases for higher $\mathrm{dB}$ output values. Furthermore, the effect of various RF maximal Doppler frequencies $\left(f_{m}=90 \mathrm{~Hz}\right.$ and $\left.f_{m}=120 \mathrm{~Hz}\right)$ and various FSO wavelengths $(\lambda=850 \mathrm{~nm}$, and $\lambda=1550 \mathrm{~nm})$ on $A F D\left(z_{t h, S D}\right)$ is also provided. 


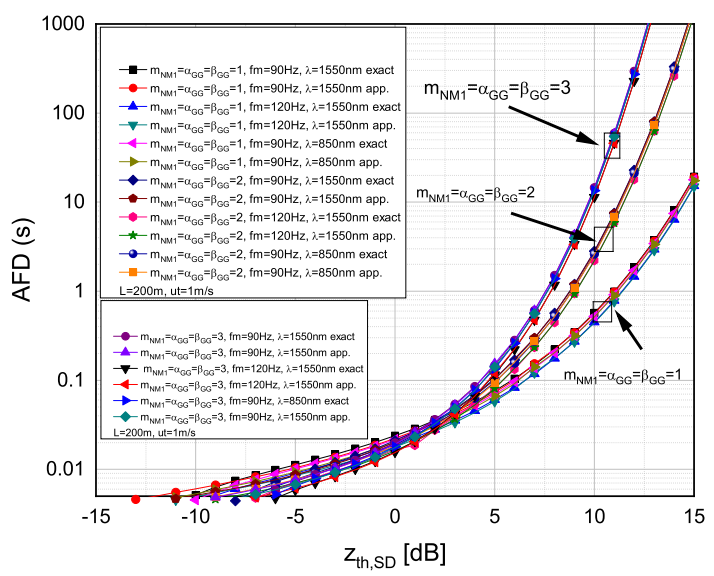

Fig. 3 Comparison of exact and approximated results for AFD for different system model parameters under different TIFC and nTIFC conditions

\section{Conclusion}

The paper considers second order statistics of the product of NM and double squared NM RVs. The provided statistical measures can be used in RF-FSO DH AF relaying systems over TIFC and nTIFC. Namely, we provide closed form approximative expressions as well as integral form exact expressions for LCR and AFD. The numerical examples show that approximations fit well with exact expressions, especially in higher signal envelope dB output regime. The system performance improvement can be reached by proposed RF-FSO system design with higher TIFC and nTIFC severities. Our future works are going to extend the proposed model to include the impact of co-channel interference in RF part of the system and pointing errors in FSO part of the system.

Acknowledgements The authors would like to acknowledge the EUIMWP COST Action 16220 project.

\section{Declarations}

\section{Funding}

This work has not been funded. 


\section{Conflicts of interest/Competing interests}

The authors declare that they have no conflict of interest or competing interests.

\section{Availability of data and material}

The authors confirm that the data supporting the findings of this study are available within the article.

\section{Code availability}

Not applicable.

\section{Authors' contributions}

The authors equally contributed to this work.

\section{References}

1. B. Talha, M. Pätzold, Channel models for mobile-to-mobile cooperative communication systems: A state of the art review, IEEE Vehicular Technology Magazine, vol. 6, no. 2, pp. 33-43 (2011)

2. J. Park, C. B. Chae, G. Yoon, Amplify-and-forward two-way relaying system over freespace optics channels, Journal of Communications and Networks, vol. 19, no. 5, pp. 481-492 (2017)

3. J. Zhang, X. Pan, G. Pan, Y. Xie, Secrecy Analysis for Multi-Relaying RF-FSO Systems With a Multi-Aperture Destination, IEEE Photonics Journal, vol. 12, no. 2, pp. 1-11 (2020)

4. Y. Zhang, J. Zhang, L. Yang, B. Ai, M. S. Alouini, On the Performance of Dual-Hop Systems over Mixed FSO/mmWave Fading Channels, IEEE Open Journal of the Communications Society, vol. 1, pp. 477-489 (2020)

5. A. Douik, H. Dahrouj, T. Y. Al-Naffouri, M. S. Alouini, Hybrid radio/free-space optical design for next generation backhaul systems, IEEE Transactions on Communications, vol. 64, no. 6, pp. 2563-2577 (2016)

6. C. Stefanovic, M. Pratesi, F. Santucci, Second Order Statistics of Mixed RF-FSO Relay Systems and its Application to Vehicular Networks, IEEE ICC'19 ONF Symposium, (2019)

7. C. Stefanovic, M. Pratesi, F. Santucci, Second Performance evaluation of cooperative communications over fading channels in vehicular networks, Second URSI Atalntic Science Radio Meeting, (2018)

8. W. Fawaz, C. Abou-Rjeily, C. Assi, UAV-aided cooperation for FSO communication systems, IEEE Communications Magazine, vol. 56, no. 1, pp. $70-75$ (2017)

9. N. Bhargav, C.R.N. da Silva, Y. J. Chun, E. J. Leonardo, S. L. Cotton, M. D. Yacoub, On the Product of Two $\kappa-\mu$ Random Variables and its Application to Double and Composite Fading Channels, IEEE Transactions on Wireless Communications, vol. 17, no. 4, pp. 2457-2470 (2018)

10. N. Milosevic, M. Stefanovic, Z. Nikolic, P. Spalevic, C. Stefanovic, Performance analysis of interference-limited mobile-to-mobile $\kappa$ - $\mu$ fading channel, Wireless Personal Communications, vol. 101, no. 3, pp. 1685-1701 (2018) 
11. N. Milosevic, C. Stefanovic, Z. Nikolic, M. Bandjur, M. Stefanovic, First-and secondorder statistics of interference-limited mobile-to- mobile Weibull fading channel, Journal of Circuits, Systems and Computers, vol. 27, no. 11, pp. 1685-1701 (2018)

12. C. Stefanovic, S. Panic, V. Mladenovic, S. Jovkovic, M. Stefanovic, Higher order statistics of cooperative mobile-to-mobile relay communications over composite fading channels, International Journal of Ad Hoc and Ubiquitous Computing, vol. 35, no. 2, pp. 61-70 (2020)

13. N. Hajri, R. Khedhiri, N. Youssef, On Selection Combining Diversity in Dual-Hop Relaying Systems Over Double Rice Channels: Fade Statistics and Performance Analysis, IEEE Access, vol. 8, pp. 72188-72203 (2020)

14. S. Li, L. Yang, D. B. Da Costa, J. Zhang, M. S. Alouini, Performance Analysis of Mixed RF-UWOC Dual-Hop Transmission Systems, IEEE Transactions on Vehicular Technology, vol. 69, no. 11, pp. 14043 - 14048 (2020)

15. W. Liu, J. Ding, J. Zheng, X. Chen, X. I. Chih-Lin, Relay-Assisted Technology in Optical Wireless Communications: A Survey, IEEE Access, vol. 8, pp. 194384 - 194409 (2020)

16. H. Liang, C. Gao, Y. Li, M. Miao, X. Li, Performance analysis of mixed MISO RF/SIMO FSO relaying systems, Optics Communications, vol. 478, p.126344 (2020)

17. S. Sharma, A. S. Madhukumar, R. Swaminathan, Performance of Dual-Hop Hybrid FSO/RF System with Pointing Errors Optimization, 2020 IEEE 91st Vehicular Technology Conference (VTC2020-Spring), pp. 1-5 (2020)

18. S. Panic, S. Mohanchenko, C. Stefanovic, M. Stefanovic, First order outage statistics of asymmetrical RF-OW dual-hop relay communications, The University ThoughtPublication in Natural Sciences, vol. 10, no. 1, pp. 57-62 (2020)

19. N. A. M. Nor, Z. Ghassemlooy, J. Bohata, P. Saxena, M. Komanec, S. Zvanovec, M. A., Bhatnagar, M. A. Khalighi, Experimental investigation of all-optical relay-assisted 10 $\mathrm{Gb} / \mathrm{s}$ FSO link over the atmospheric turbulence channel, Journal of Lightwave Technology, vol. 35, no. 1, pp. 45-53 (2016)

20. H. T. Yura, S. G. Hanson, Mean level signal crossing rate for an arbitrary stochastic process, J. Opt. Soc. Am., vol. 27, no. 4, pp. 797-807 (2010)

21. A. Jurado-Navas, J. M. Garrido Balsells, M Castillo-Vazquez, A. Puerta-Notario, I. T. Monroy, J. J. V. Olmos, Fade statistics of M-turbulent optical links, EURASIP Journal on Wireless Communications and Networking, 2017:112 (2017), DOI:10.1186/s136380170898-z.

22. D. Stefanovic, C. Stefanovic, D. Djosic, D. Milic, D. Rancic, M. Stefanovic, LCR of the Ratio of the Product of Two Squared Nakagami-m Random Processes and Its Application to Wireless 2019 18th IEEE International Symposium INFOTEH-JAHORINA (INFOTEH), pp. 1-4 (2019)

23. C. B. Issaid, M. S. Alouini, Level Crossing Rate and Average Outage Duration of Free Space Optical Links, IEEE Transactions on Communications, vol. 67, no. 9, pp. 6234 $6242(2019)$

24. F. S. Vetelino, C. Young, L. Andrews, Fade statistics and aperture averaging for Gaussian beam waves in moderate-to-strong turbulence, Applied optics, vol. 46, no. 18, pp. 3780-3789 (2007)

25. H. K. Kim, T. Higashino, K. Tsukamoto, S. Komaki, Optical fading analysis considering spectrum of optical scintillation in terrestrial free-space optical channel, IEEE International Conference on Space Optical Systems and Applications, pp. 58-66 (2011)

26. L. G. Stüber, Principles of mobile communication. Kluwer Academic, Mass, USA (1996)

27. S. Suljovic, D. Milic, S. Panic, C. Stefanovic, M. Stefanovic, Level crossing rate of macro diversity reception in composite Nakagami-m and Gamma fading environment with interference, vol. 102, p.102758 (2020), DOI:10.1016/j.dsp.2020.102758

28. C. Stefanovic, B. Jaksic, P. Spalevic, S. Panic, Z. Trajcevski, Performance analysis of selection combining over correlated Nakagami-m fading channels with constant correlation model for desired signal and cochannel interference, Radioengineering, vol. 22, no. 4, pp. 1176-1181 (2013)

29. S. Al-Ahmadi, The gamma-gamma signal fading model: A survey, IEEE Antennas and Propagation Magazine, vol. 56, no. 5, pp. 245-260 (2014) 
30. M. Petkovic, N. Zdravkovic, C. Stefanovic, G. Djordjevic, Performance analysis of SIM-FSO system over Gamma-Gamma atmospheric channel, XLIX International Scientific Conference on Information, Communication and Energy Systems and Technologies ICEST 2014, vol. 1, pp. 19-22 (2014)

31. C. K. Datsikas, K. P. Peppas, N. C. Sagias, G. S. Tombras, Serial free-space optical relaying communications over gamma-gamma atmospheric turbulence channels, IEEE/OSA Journal of Optical Communications and Networking, vol. 2, no. 8, pp. 576-586 (2010)

32. O. S. Badarneh, S. Muhaidat, P. C. Sofotasios, S. L. Cotton, K. Rabie, D. B. da Costa The N* Fisher-Snedecor F cascaded fading model, 14th International Conference on Wireless and Mobile Computing, Networking and Communications (WiMob), pp. 1-7 (2018)

33. G. K. Karagiannidis, N. C. Sagias, P. T. Mathiopoulos, N* Nakagami: A Novel Stochastic Model for Cascaded Fading Channels, IEEE Transactions on Communications, vol. 55, no. 8, pp. 1453-1458 (2007)

34. P. S. Bithas, A. G. Kanatas, D. B. da Costa, P. K. Upadhyay, U. S. Dias, On the double-generalized gamma statistics and their application to the performance analysis of V2V communications, IEEE Transactions on Communications, vol. 66, no. 1, pp. 448-460 (2018)

35. H. Al-Hmood, R. S. Abbas, H. S. Al-Raweshidy, Ratio of Products of Mixture Gamma Variates with Applications to Wireless Communications, IEEE Transactions on Communications, arXiv preprint arXiv:2007.10826 (2020)

36. A. Stankovic, C. Stefanovic, N. Sekulovic, Z. Popovic, M. Stefanovic, The distribution of minimum of ratios of two random variables and its application in analysis of multi-hop systems, Radioengineering, vol. 21, no. 4, pp. 1156-1162 (2012)

37. A. Matovic, E. Mekic, N. Sekulovic, M. Stefanovic, M. Matovic, C. Stefanovic, The distribution of the ratio of the products of two independent - variates and its application in the performance analysis of relaying communication systems, Mathematical Problems in Engineering, vvol. 2013, Article ID 147106, doi:10.1155/2013/147106 (2013)

38. Z. Hadzi-Velkov, N. Zlatanov, G. K. Karagiannidis, On the second order statistics of the multihop rayleigh fading channel, IEEE Transactions on Communications, vol. 57, no. 6, pp 1815 - 1823 (2009)

39. C. Stefanovic, D. Djosic, S. Panic, D. Milic, M. Stefanovic, A Framework for Statistical Channel Modeling in 5G Wireless Communication Systems. 5G Multimedia communications: Technology, multiservices, deployment, IEEE Transactions on Communications, CRC Press 2020, pp. 31-54 (2020)

40. I. S. Gradshteyn, I. M. Ryzhik, Table of Integrals, Series, and Products, IEEE Transactions on Communications, New York: Academic, 6th edition (2000)

41. L. C. Andrews and R. L. Phillips, Laser Beam Propagation Through Random Media SPIE Press, 2nd edition (2005) 